\title{
Transport of ions through an elliptic ion-selective membrane
}

\author{
Jyh-Ping Hsu*, Bo-Tau Liu \\ Department of Chemical Engineering, National Taiwan University, Taipei 10617, Taiwan, ROC
}

Received 27 August 1997; received in revised form 13 November 1997; accepted 6 December 1997

\begin{abstract}
The rate of transport of ions through an annular ion-selective membrane having an elliptic cross section is analyzed. This is a generalization to a hollow-fiber type of device, and represents a wide class of membranes which are capable of providing a large (surface area/volume) ratio for ion transport. The Nernst-Plank equation governing the transport of ions is solved numerically. The results obtained reveal that, if the concentration of fixed charge is high, its current efficiency is insensitive to fixed charge distribution, and the local electroneutrality can be assumed. On the other hand, if the concentration of fixed charge is low, the distribution of fixed charge becomes significant, and assuming local electroneutrality can be inappropriate. In general, the higher the average concentration (or the greater the total amount) of fixed charge of a membrane, the higher its current efficiency. We show that the results for planar and cylindrical membranes can be recovered from the present model. (C) 1998 Elsevier Science B.V.
\end{abstract}

\section{Introduction}

The interest in the applications of various types of membrane is continuously growing in the past few decades. One of these applications is the separation of ions $[1,3]$. Here, a membrane bearing dissociable functional groups is immersed in an electrolyte solution, and an electric field applied. The dissociation of the functional groups results in a charged membrane, which has the effect of repelling coions and attracting counterions. Therefore counterions tend to stay in the liquid phase on one side of the membrane, and coions tend to stay in the liquid phase on the other side. The applied electric field provides the driving force for the transport of ions through the membrane. Apparently, the performance of a membrane is closely related to its

\footnotetext{
*Corresponding author. Fax: 886-2-3623040; e-mail: t8504009@ccms.ntu.edu.tw
}

physicochemical properties. This includes, for instance, the nature of a membrane (cation-selective or anion-selective), its shape, and the concentration as well as the distribution of dissociable functional groups. Relevant studies concerning the influence of these factors on the performance of a membrane are ample in the literature. Reiss and Bassignana [4], for example, concluded that the current efficiency of a membrane increases with the degree of nonuniformity of fixed charge. Further analysis, however, revealed that this effect can be negative [5]. Sokirko et al. [6] took the effect of applied current density into account. It was found that the performance of a membrane increases with the inhomogeneity of fixed charge distribution, and the degree of improvement correlates with the applied current density.

Previous efforts are almost always limited to the case of planar membranes. Relative little attention was paid to other possible alternatives. In a recent study, 
Hsu and Yang [7] proposed using a cylindrical membrane. This type of device is capable of providing a large (surface area/volume) ratio, thereby yielding a large transfer rate for ions in a limited space. In practice, a hollow-fiber type of device can be designed which comprises numerous parallel annular membranes each has a small diameter [8]. For the same total surface area for ion transport, it is superior to an array of planar membranes, for the former occupies much less space than the latter.

In this study, the analysis of Hsu and Yang [7] is extended to a more general case that a membrane has an elliptic cross section. Although this is a purely novel device at the present stage, it provides a flexible basis for design purposes. By varying the parameters of an elliptic coordinates, various shapes can be described. In other words, it is capable of simulating a wide class of annular membranes. Another potential usage of the present analysis is that the deformation of an annular membrane as a response to the variation in the operating conditions can be modeled. For instance, the cross section of a cylindrical membrane may become elliptic under the hydrodynamic force exerted by the surrounding fluid. It should be pointed out that although a hollow-fiber type of device has been used widely in the area of biotechnology and bioengineering, its potential usage in the separation of ions through an ion-selective membrane has not been discussed in the literature, and a detailed analysis on its behavior and performance is highly desirable.

\section{Modeling}

By referring to Fig. 1, we consider an annular ionselective membrane having an elliptic cross section immersed in an electrolyte solution. An electrical potential difference is applied across the membrane. Without loss of generality, we consider a cationselective membrane; i.e., the fixed charges carried by membrane are negative. In this case, cations migrate from the inner liquid phase, pass through the membrane, and then move to the outer liquid phase; the anions move in the inverse direction. The bulk concentrations of ions inside and outside membrane are denoted by $C_{0}$ and $C_{\mathrm{f}}$, respectively. We assume that the transfer of ions can be described by the Nernst-Planck equation [2]. For symmetric electrolytes, the flux of ion species $j, \vec{J}_{j}$, is

$\vec{J}_{j}=-D_{j}\left[\vec{\nabla} C_{j}+(-1)^{j+l} \frac{Z_{j} F}{R T} C_{j} \vec{\nabla} \psi\right]$

where $\psi$ denotes the electrical potential, $\vec{\nabla}$ the gradient operator, $j$ an index, $D_{j}$ and $C_{j}$ the diffusivity and the concentration of ion species $j$ ( $j=1$ for cation, $j=2$ for anion), $F$ the Faraday constant, $Z_{j}$ the valence of ion species $j, R$ and $T$ the gas constant and the absolute temperature, respectively. For simplicity, we assume that $D_{1}=D_{2}=D$, and $Z_{1}=Z_{2}=Z$. The electrical potential is governed by the Poisson equation [9]

$$
\nabla^{2} \psi=-\frac{Z F}{\epsilon}\left[C_{1}-C_{2}-C_{3}\right]
$$

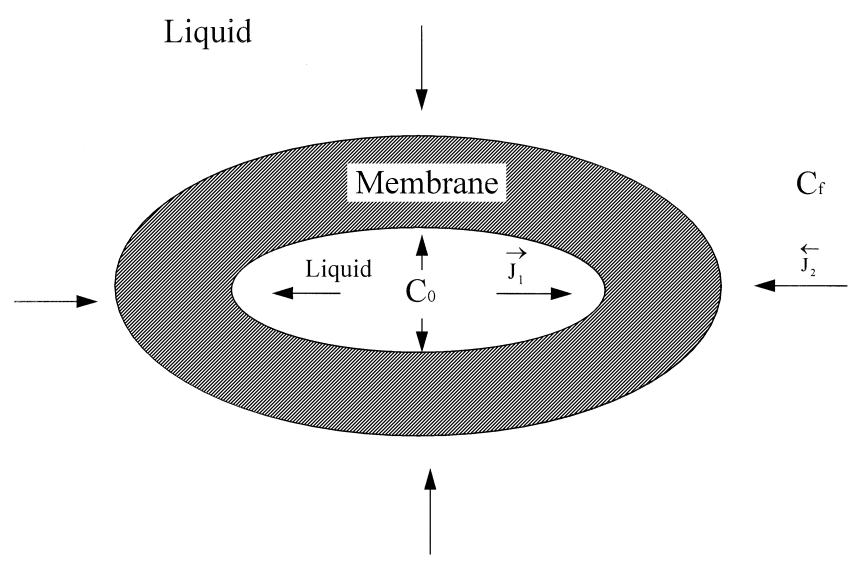

Fig. 1. A schematic representation of the system under consideration. The membrane carries negative fixed charges. $\vec{J}_{1}$ and $\vec{J}_{2}$ are the fluxes of cation and anion, respectively. $C_{\mathrm{o}}$ and $C_{\mathrm{f}}$ are the bulk liquid concentrations of ions inside and outside membrane, respectively. 


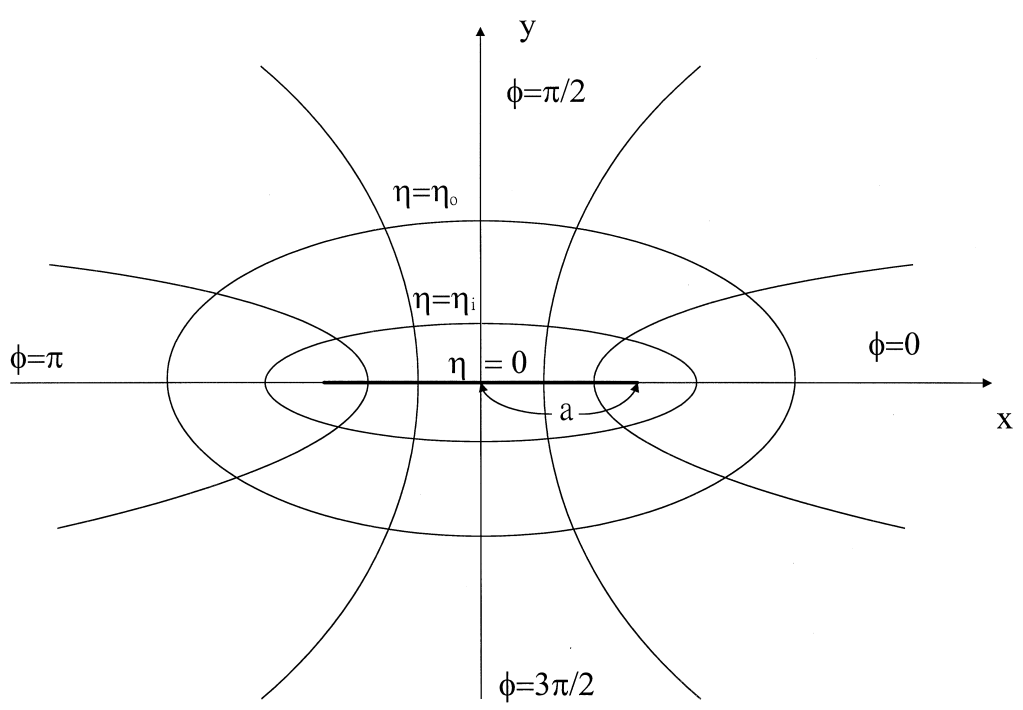

Fig. 2. A schematic representation of the coordinates adopted.

where $C_{3}$ is the concentration of fixed charge in membrane. The corresponding boundary conditions are assumed as

$\psi=\psi_{\mathrm{i}}$ at inner liquid-membrane interface

$\psi=\psi_{\mathrm{o}}$ at outer liquid-membrane interface

The current density, $\vec{I}$, can be expressed by

$\vec{I}=F Z\left(\vec{J}_{1}-\vec{J}_{2}\right)$

The coordinates adopted in the present analysis are illustrated in Fig. 2. Here, $\eta$ and $\phi$ are, respectively, the radial and the angle coordinates, and $a$ the distance between the focus and the center of an ellipse. The inner and the outer liquid-membrane interfaces are represented by $\eta=\eta_{\mathrm{i}}$ and $\eta=\eta_{\mathrm{o}}$, respectively. The metric coefficients for the coordinates adopted are [10]

$$
\begin{aligned}
& g_{11}=g_{22}=a^{2}\left[\cos ^{2} \eta-\cos ^{2} \phi\right] \\
& g_{33}=1
\end{aligned}
$$

Note that, since these coefficients are functions of $\eta$ and $\phi$, so are the fluxes of ions. Suppose that the system is at the steady state. Taking the divergence on both sides of Eq. (1) yields

$$
\begin{aligned}
& \nabla^{2} p+\nabla p \cdot \nabla \bar{\psi}+p \nabla^{2} \bar{\psi}=0 \\
& \nabla^{2} n-\nabla n \cdot \nabla \bar{\psi}-n \nabla^{2} \bar{\psi}=0
\end{aligned}
$$

where

$$
\begin{aligned}
& \bar{\psi}=\frac{Z F \psi}{R T} \\
& p=C_{1} / C_{0} \\
& n=C_{2} / C_{0}
\end{aligned}
$$

In these expressions, $y$ is the scaled electrical potential, and $p$ and $n$ the scaled concentrations of cations and anions, respectively. Eq. (2) can be rewritten in the scaled form

$\frac{\partial^{2} \bar{\psi}}{\partial \eta^{2}}+\frac{\partial^{2} \bar{\psi}}{\partial \phi^{2}}=-\frac{\chi^{2}}{2} g_{11}[p-n-q]$

where

$$
\begin{aligned}
& q=C_{3} / C_{0} \\
& \chi=\kappa a \\
& \kappa^{2}=2 F^{2} Z^{2} C_{0} / \epsilon R T
\end{aligned}
$$

Here, $q$ is the scaled concentration of fixed charges, $\chi$ the scaled distance between the focus and the center of an ellipse, and the reciprocal Debye length. Suppose that Donnan equilibrium can be assumed at the liquid-membrane interfaces $[11,12]$. Then, it can be shown that

$p(0)=\frac{q(0)}{2}+\sqrt{\frac{q(0)^{2}}{4}+1}$ 
$n(0)=1 / p(0)$

$p(1)=\frac{q(1)}{2}+\sqrt{\frac{q(1)^{2}}{4}+\gamma^{2}}$

$n(1)=\gamma^{2} / p(1)$

In these expressions, $\gamma=C_{\mathrm{f}} / C_{0}, p(0)$ and $n(0)$ are the values of $p$ and $n$ at the inner liquid-membrane interface $\left(\eta=\eta_{\mathrm{i}}\right)$, and $p(1)$ and $n(1)$ are those at the outer liquid-membrane interface $\left(\eta=\eta_{\mathrm{o}}\right)$.

Solving Eqs. (5)-(7) subject to the boundary conditions, Eqs. (2a) and (2b) and Eqs. (8a)-(8d), yields the distributions of electrical potential and ions. In general, a numerical scheme is inevitable.

\section{Results}

Two classes of fixed charge distribution are adopted to illustrate the behavior of the system under consideration: linear and sinusoidal. A general expression for the former is

$q=\alpha_{\mathrm{L}}+\delta_{\mathrm{L}} \xi$

where

$\xi=\frac{\eta-\eta_{\mathrm{i}}}{\eta_{\mathrm{o}}-\eta_{\mathrm{i}}}$

For comparison purposes, we assume that the average concentration (or total amount) of fixed charge is constant. In this case $\alpha_{\mathrm{L}}$ and $\delta_{\mathrm{L}}$ need to satisfy

$\alpha_{\mathrm{L}}=\bar{q}+\delta_{\mathrm{L}} \frac{\sigma_{\mathrm{L}}}{S}$

where

$$
\begin{aligned}
\sigma_{\mathrm{L}}= & \frac{\pi}{4\left(\eta_{\mathrm{o}}-\eta_{\mathrm{i}}\right)}\left[\cosh \left(2 \eta_{\mathrm{i}}\right)-\cosh \left(2 \eta_{\mathrm{o}}\right)\right. \\
& \left.+2\left(\eta_{\mathrm{o}}-\eta_{\mathrm{i}}\right) \sinh \left(2 \eta_{\mathrm{o}}\right)\right] \\
S= & \frac{\pi}{2}\left[\sinh \left(2 \eta_{\mathrm{o}}\right)-\sinh \left(2 \eta_{\mathrm{i}}\right)\right]
\end{aligned}
$$

Here, $\bar{q}$ is the scaled average concentration of fixed charge, and $S$ the scaled cross sectional area of a membrane. The total amount of fixed charge is $\bar{q} S W, W$ being the length of the membrane. Similarly, for a sinusoidal fixed charge distribution, we have

$$
q=\alpha_{\mathrm{s}}+\delta_{\mathrm{s}} \sin (2 \pi \xi)
$$

with

$$
\begin{aligned}
& \alpha_{\mathrm{s}}=\bar{q}+\delta_{\mathrm{s}} \frac{\sigma_{\mathrm{s}}}{S} \\
& \sigma_{\mathrm{s}}=\frac{\pi^{2}\left(\eta_{\mathrm{i}}-\eta_{\mathrm{o}}\right)\left[\cosh \left(2 \eta_{\mathrm{o}}\right)-\cosh \left(2 \eta_{\mathrm{i}}\right)\right]}{2\left[\left(\eta_{\mathrm{o}}-\eta_{\mathrm{i}}\right)^{2}+\pi^{2}\right]}
\end{aligned}
$$

Fig. 3 shows the contours of the concentrations of ions over the $\phi-\eta$ domain in the membrane region for a higher fixed charge concentration, those for a lower fixed charge concentration is illustrated in Fig. 4. Here, we assume that fixed charge distribution is a function of the radial variable, $\eta$, only. In this case, the distributions of ions are independent of the angle variable, $\phi$, as can be seen from Figs. 3 and 4 .

Note that, since $\vec{J}_{j}$ is a function of both $\eta$ and $\phi$, only the averaged performance of a membrane can be evaluated. To this end, we calculate the rate of transfer of ion species $j$ across the liquid-membrane interfaces, $J_{j}$, by

$$
J_{j}=\int_{0}^{2 \pi} \int_{0}^{w} \vec{J}_{j} \cdot\left(\vec{e}_{\phi} \times \vec{e}_{z}\right) \mathrm{d} \phi \mathrm{d} z
$$

where $\vec{e}_{\phi}$ and $\vec{e}_{z}$ are the unit base vectors in $\phi$ and $z$ directions, respectively. $J_{j}$ is constant at the steady state. If the fixed charge concentration is a function of the radial variable only, integrating Eq. (1) over the surface represented by $\eta=$ constant gives

$-\frac{\mathrm{d} p}{\mathrm{~d} \xi}-p \frac{\mathrm{d} Y}{\mathrm{~d} \xi}=h$
$-\frac{\mathrm{d} n}{\mathrm{~d} \xi}+n \frac{\mathrm{d} Y}{\mathrm{~d} \xi}=g$

where

$$
\begin{aligned}
& Y=\frac{1}{2 \pi} \int_{0}^{2 \pi} \bar{\psi} \mathrm{d} \phi \\
& h=\frac{J_{1}\left(\eta_{\mathrm{o}}-\eta_{\mathrm{i}}\right)}{2 \pi D C_{0} w} \\
& g=\frac{J_{2}\left(\eta_{\mathrm{o}}-\eta_{\mathrm{i}}\right)}{2 \pi D C_{0} w}
\end{aligned}
$$

Note that Eqs. (12) and (13) have the same form as that for the case of planar membranes [5]. This means that, if the scaled variables are defined appropriately, 


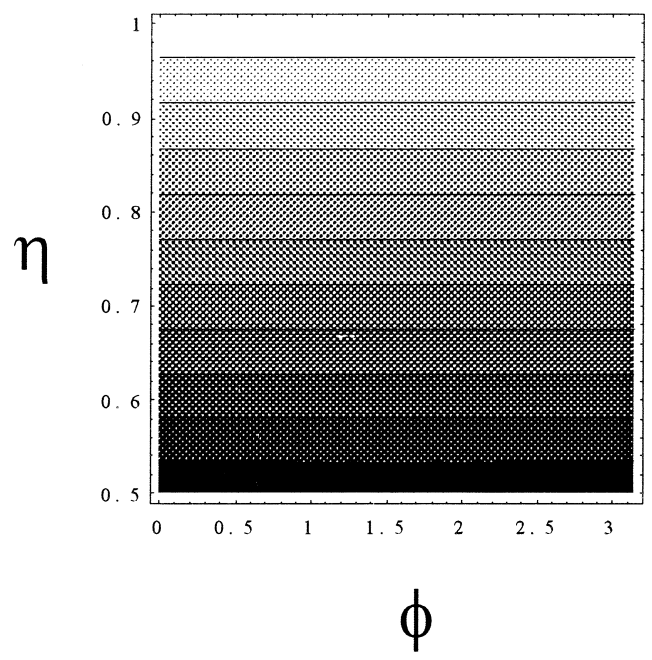

(a)

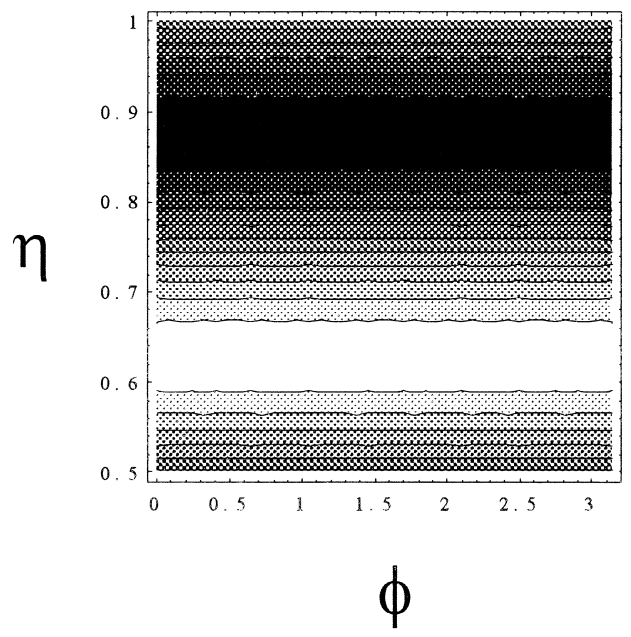

(c)

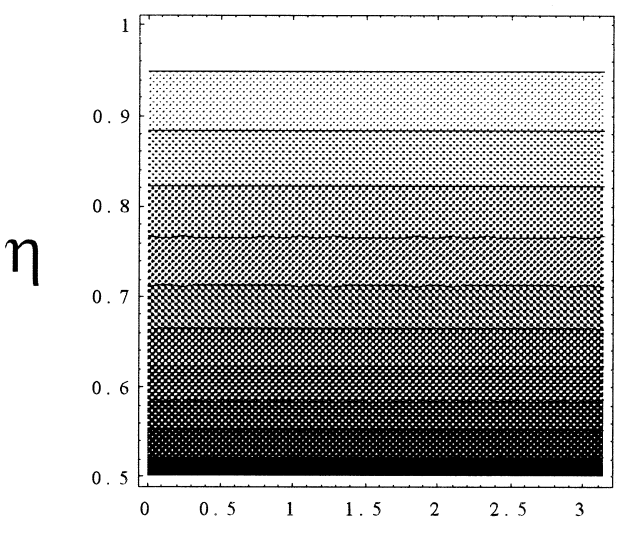

$\phi$

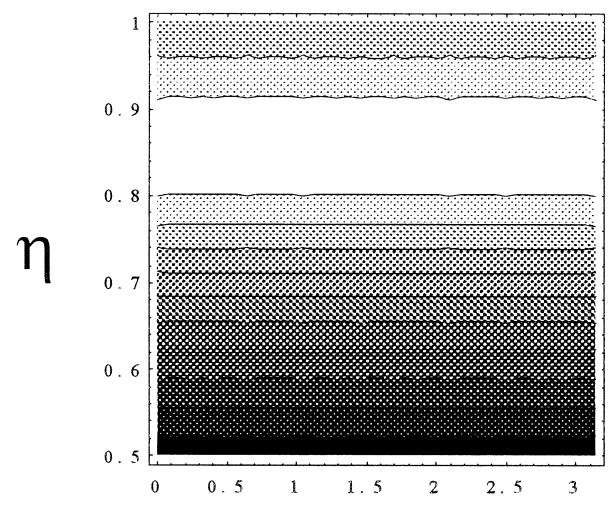

$\phi$

Fig. 3. Contours of the concentrations of ions over the domain in the membrane region. The level of concentration is proportional to the darkness of the area. Cation: (a) and (c); anion: (b) and (d). The fixed charge distribution is linear in (a) and (b) with $\delta_{\mathrm{L}}=4$, and sinusoidal in (c) and (d) with $\delta_{\mathrm{s}}=2$. Key: $\bar{q}=10, \eta_{\mathrm{i}}=0.5, \eta_{\mathrm{o}}=1, \lambda^{2}=4000, \gamma=2, \Delta \bar{\psi}=2, \Delta \bar{\psi}$ being the scaled electrical potential drop across membrane, and the average concentration of fixed charge is the same in each case. 

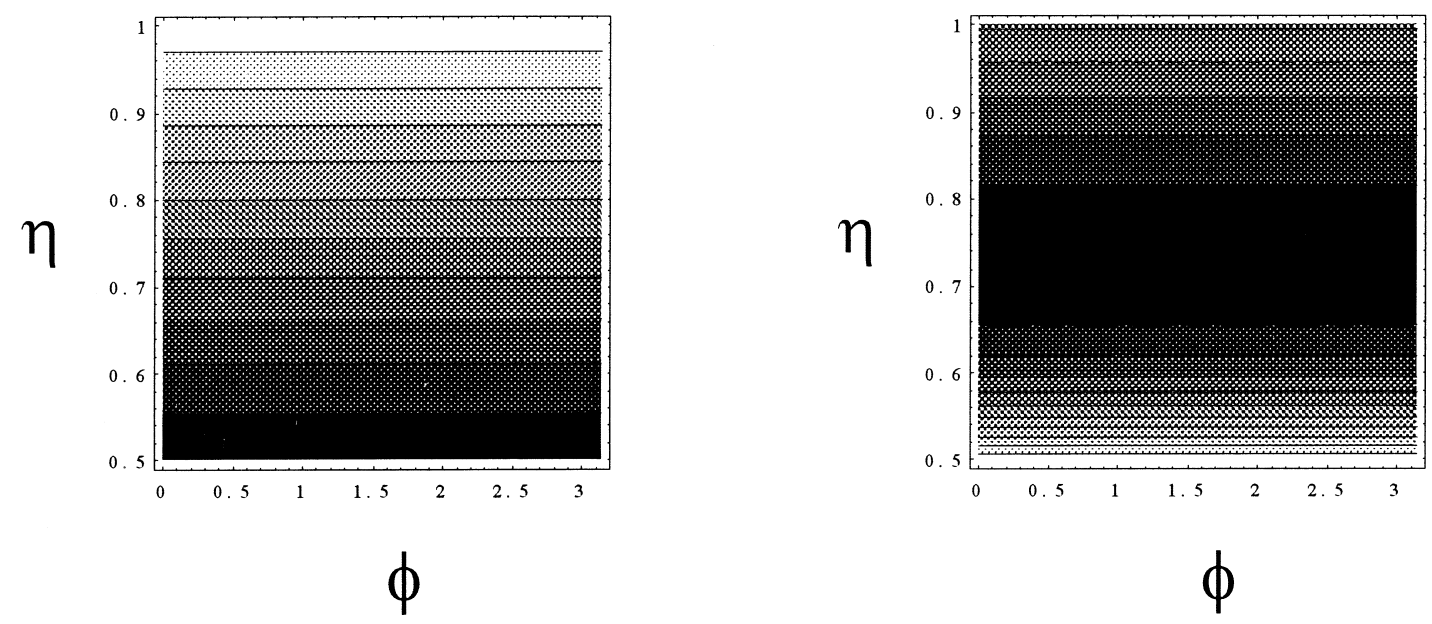

(a)
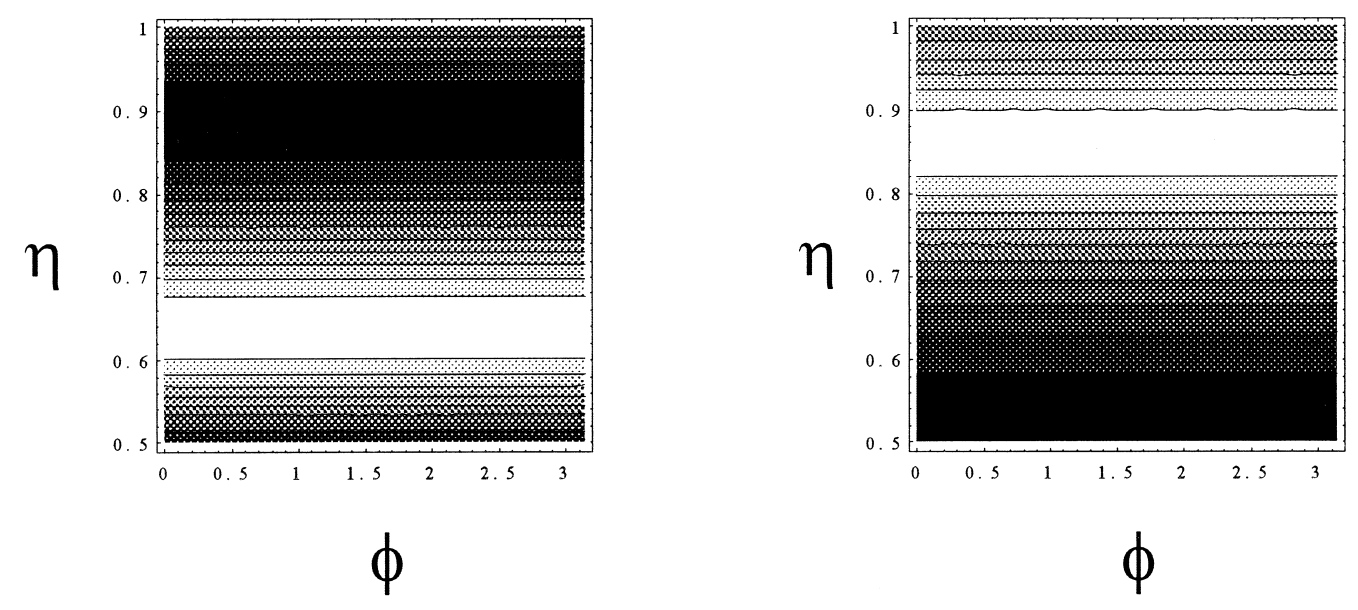

$\phi$

(c)

(d)

Fig. 4. Contours of the concentrations of ions over the domain in the membrane region. The symbols are the same as those of Fig. 3. Key: same as Fig. 3, except that $\bar{q}=2$ and $\Delta \bar{\psi}=5$. 
the results for planar membranes can be deduced from the present model.

\subsection{Special cases}

Two special cases of the present model deserves further discussion.

\subsubsection{Cylindrical membranes}

The surface described by $\eta=$ constant can also be expressed as [10]

$\left(\frac{x}{a \cosh \eta}\right)^{2}+\left(\frac{y}{a \sinh \eta}\right)^{2}=1$

where $x$ and $y$ are the Cartesian coordinates. If $\eta$ is sufficiently large, Eq. (14) becomes

$x^{2}+y^{2}=\left(\frac{a}{2} \exp (\eta)\right)^{2}$

This portrays a circle. In other words, the results for cylindrical membranes [7] can be recovered from the present elliptic membrane by assuming a large $\eta_{\mathrm{i}}$. Furthermore, if $\eta_{\mathrm{i}}$ is large and $\eta_{\mathrm{o}}-\eta_{\mathrm{i}}$ is small, then the present elliptic membrane can be treated as a planar membrane.

\subsubsection{Local electroneutrality}

If the condition of local electroneutrality applies [13], the right-hand side of Eq. (7) vanishes, i.e.,

$p-n-q=0$

In this case, Eqs. (12),(13) and (16) lead to

$\frac{\mathrm{d} p}{\mathrm{~d} \xi}=\frac{p \frac{\mathrm{d} q}{\mathrm{~d} \xi}-p(2 h-i)+q \cdot h}{2 p-q}$

$\frac{\mathrm{d} n}{\mathrm{~d} \xi}=-\frac{n \frac{\mathrm{d} q}{\mathrm{~d} \xi}+n(2 g+i)+q \cdot g}{2 n+q}$

where the scaled current, $i$, is defined by

$i=\frac{I\left(\eta_{\mathrm{o}}-\eta_{\mathrm{i}}\right)}{2 \pi F Z D C_{0} W}$

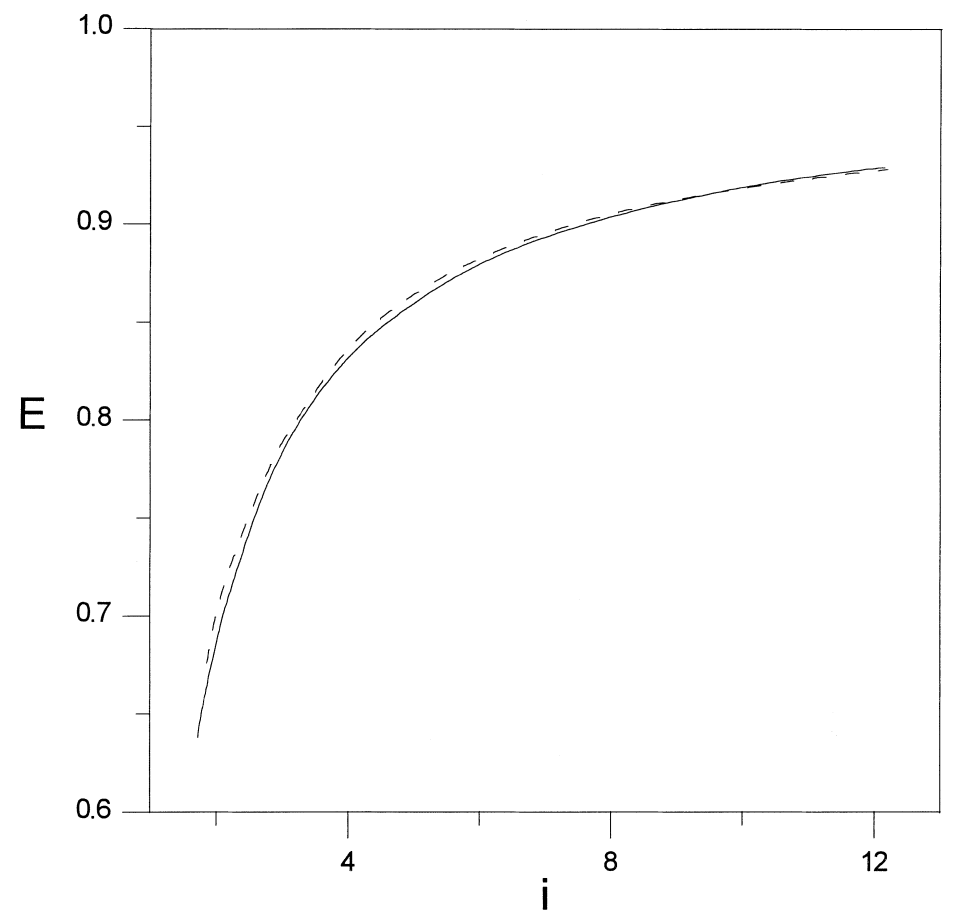

Fig. 5. Variation in the current efficiency $E$ as a function of the scaled current $i$. Solid line: linear fixed charge distribution with $\delta_{\mathrm{L}}=4$; dashed line: sinusoidal fixed charge distribution with $\delta_{\mathrm{s}}=2$. Key: $\bar{q}=10, \eta_{\mathrm{i}}=0.5, \eta_{\mathrm{o}}=1, \gamma=2$, and the average concentration of fixed charge is the same in each case. 


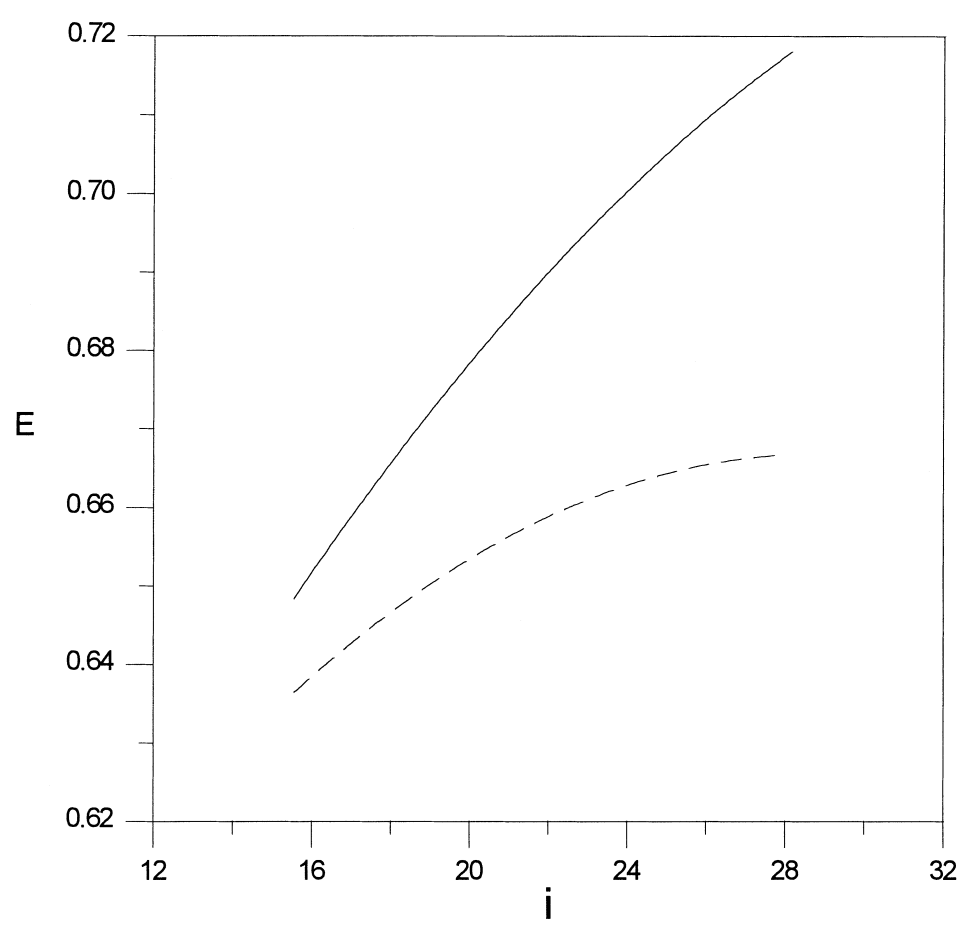

Fig. 6. Variation in the current efficiency $E$ as a function of the scaled current $i$. (a): linear fixed charge distribution with $\delta_{\mathrm{L}}=4$; (b): sinusoidal fixed charge distribution with $\delta_{\mathrm{s}}=2$. Key: same as Fig. 5, except that $\bar{q}=2$.

with $I=F Z\left(J_{1}-J_{2}\right), I$ being the current. Note that in the present case, solving either Eq. (17) for $p$ or Eq. (18) for $n$ is sufficient; if $p$ (or $n$ ) is solved, then $n$ (or $p$ ) can be determined by Eq. (16). In other words, under the condition of local electroneutrality, solving the Poisson equation, Eq. (7), becomes unnecessary. The boundary conditions for electrical potential, Eqs. (2a) and $(2 b)$, however, need to be replaced by the specified current. Solving Eq. (17) or Eq. (18) analytically is nontrivial, in general. Here, an iterative procedure can be employed [14]. For a given $i$, an arbitrary $h$ is guessed, and Eq. (17) solved subject to Eq. (8a). The value of $p$ at the outer liquid-membrane interface, $p(1)$, is calculated, and the result compared with Eq. (8c). If the agreement is satisfactory, the guessed $h$ is acceptable. Otherwise, another $h$ is assumed, and the numerical procedure repeated.

Fig. 5 shows the variation in the current efficiency, $E$, as a function of the scaled current, $i$, for the case of a higher fixed charge concentration; that for a lower fixed charge concentration is presented in Fig. 6 . $E$ is defined as
$E=\frac{|h|}{|h|+|g|}$

As can be seen from Fig. 5, if the concentration of fixed charge is high, $E$ is independent of the type of fixed charge distribution. On the other hand, if it is low, the distribution becomes significant, as suggested by Fig. 6 . A comparison between Figs. 5 and 6 shows that $E$ increases with the average concentration (or total amount) of fixed charge. For the same total amount of fixed charge, the linear fixed charge distribution yields a higher current efficiency than the sinusoidal fixed charge distribution does.

The variation in the scaled net charge, $(p-n-q)$, as a function of the radial variable $\eta$, for a higher fixed charge concentration is illustrated in Fig. 7, and that for a lower fixed charge concentration shown in Fig. 8. Fig. 7 reveals that the condition of local electroneutrality is not satisfied, in general. For the case of linear fixed charge distribution, the net charge in the membrane phase is negative, and the deviation from local electroneutrality is of the order of $10^{-3}$. It fluctuates 

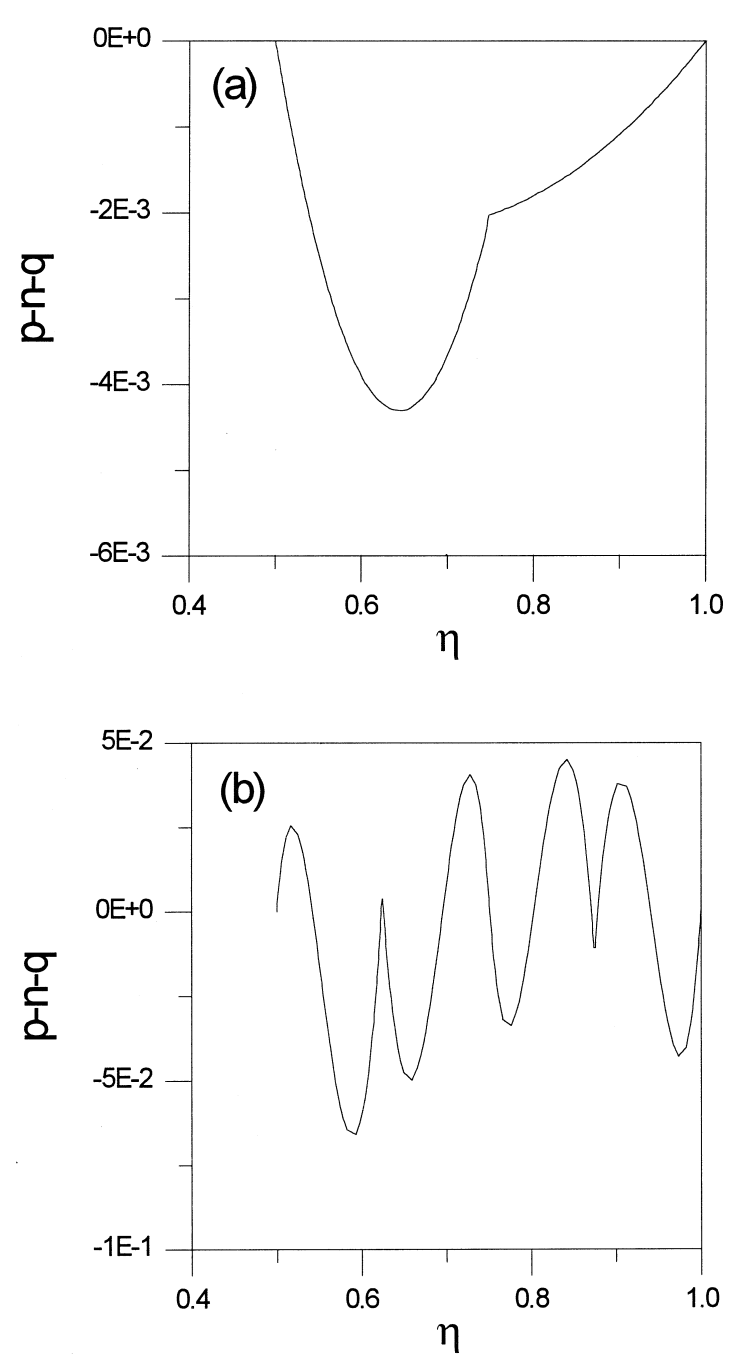

Fig. 7. Variation in the scaled net charges $(p-n-q)$ as a function of radial variable $\eta$ at $\phi=0$. (a): linear fixed charge distribution with $\delta_{\mathrm{L}}=4$; (b): sinusoidal fixed charge distribution with $\delta_{\mathrm{s}}=2$. Key: same as Fig. 3 .

around zero for the case of sinusoidal fixed charge distribution, and the deviation from local electroneutrality is of the order of $10^{-2}$. The fluctuation may be due to the behavior of a sinusoidal function and the nonlinear nature of the system of equations, Eqs. (5)(7). The deviation from local electroneutrality becomes more serious if the concentration of fixed charge is low, as suggested by Fig. 8; the deviation is of the order of $10^{-2}$ for the case of linear fixed charge distribution, and $10^{-1}$ for the case of sinusoidal fixed
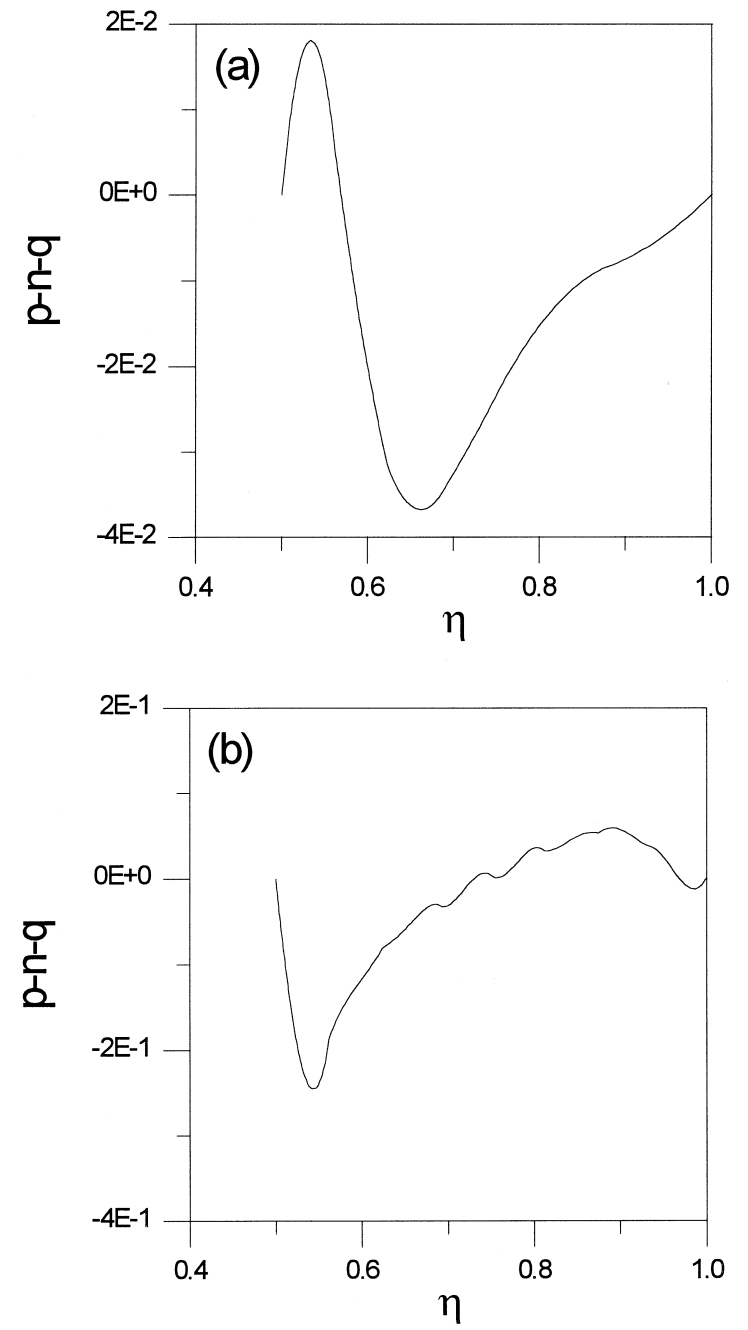

Fig. 8. Variation in the scaled net charge $(p-n-q)$ as a function of radial variable $\eta$ at $\phi=0$. Solid line: linear fixed charge distribution with $\delta_{\mathrm{L}}=4$; dashed line: sinusoidal fixed charge distribution with $\delta_{\mathrm{s}}=2$. The average concentration of fixed charge is the same in each case. Key: same as Fig. 4.

charge distribution. A comparison between Fig. 7(b) and Fig. 8(b) reveals that the fluctuation behavior of the net charge for the case of sinusoidal fixed charge distribution is less appreciable if the concentration of fixed charge is low.

\section{Conclusion}

In summary, the behavior of an annular membrane having an elliptic cross section is examined. It is 
capable of simulating a wide class of annular membranes. The elliptic membrane is a more realistic design than an exact cylindrical membrane, since the deformation of the latter may be significant in practice. The effects of the properties of a membrane on its performance are found to be similar to those for planar membranes. We show that planar membranes and cylindrical membranes can be recovered as the special cases of the present design.

\section{List of symbols}

distance between the focus and the center of an ellipse $(\mathrm{m})$

$C_{0}$ bulk concentration of ions inside membrane $\left(\mathrm{mol} / \mathrm{m}^{3}\right)$ concentration of cation $\left(\mathrm{mol} / \mathrm{m}^{3}\right)$ concentration of anion $\left(\mathrm{mol} / \mathrm{m}^{3}\right)$ concentration of fixed charge in membrane $\left(\mathrm{mol} / \mathrm{m}^{3}\right)$ bulk concentration of ions outside membrane $\left(\mathrm{mol} / \mathrm{m}^{3}\right)$ diffusivity of ion $\left(\mathrm{m}^{2} / \mathrm{s}\right)$ diffusivity of ion species $j\left(\mathrm{~m}^{2} / \mathrm{s}\right)$ unit base vector $(-)$ current efficiency $(-)$ Faraday constant (Coulomb/mol) metric coefficient $\left(\mathrm{m}^{2}\right)$ scaled rate of transfer of cation (-) scaled rate of transfer of anion (-) current (Coulomb/s) current density (Coulomb/s/m²) scaled current $(-)$ rate of transfer of cation $(\mathrm{mol} / \mathrm{s})$ rate of transfer of anion $(\mathrm{mol} / \mathrm{s})$ flux of cation $\left(\mathrm{mol} / \mathrm{s} / \mathrm{m}^{2}\right)$ flux of anion $\left(\mathrm{mol} / \mathrm{s} / \mathrm{m}^{2}\right)$ scaled concentration of anion (-) scaled concentration of cation (-) scaled average concentration of fixed charge $(-)$ scaled concentration of fixed charge (-) gas constant $(\mathrm{J} / \mathrm{mol} / \mathrm{K})$ scaled cross sectional area of a membrane $(-)$ temperature $(\mathrm{K})$ length of a membrane (m)

\begin{tabular}{|c|c|}
\hline$x, y, z$ & coordinate variables $(\mathrm{m})$ \\
\hline Z & valence of ion $(-)$ \\
\hline$Z_{j}$ & valence of ion species $j(-)$ \\
\hline$\alpha_{\mathrm{L}}$ & $\begin{array}{l}\text { parameter of linear fixed charge distribu- } \\
\text { tion }(-)\end{array}$ \\
\hline$\alpha_{\mathrm{s}}$ & $\begin{array}{l}\text { parameter of sinusoidal fixed charge dis- } \\
\text { tribution }(-)\end{array}$ \\
\hline$\gamma$ & $C_{\mathrm{f}} / C_{0}(-)$ \\
\hline$\delta_{\mathrm{L}}$ & $\begin{array}{l}\text { parameter of linear fixed charge dis- } \\
\text { tribution }(-)\end{array}$ \\
\hline$\delta_{\mathrm{s}}$ & $\begin{array}{l}\text { parameter of sinusoidal fixed charge dis- } \\
\text { tribution }(-)\end{array}$ \\
\hline$\epsilon$ & dielectric constant $\left(\right.$ Coulomb $\left.^{2} / \mathrm{J} / \mathrm{m}\right)$ \\
\hline$\eta$ & radial coordinate $(-)$ \\
\hline$\eta_{\mathrm{i}}$ & $\begin{array}{l}\eta=\eta_{\mathrm{i}} \text { denotes the inner liquid-membrane } \\
\text { interface }(-)\end{array}$ \\
\hline$\eta_{\mathrm{o}}$ & $\begin{array}{l}\eta=\eta_{\mathrm{o}} \text { denotes the outer liquid-membrane } \\
\text { interface }(-)\end{array}$ \\
\hline$\kappa$ & reciprocal Debye length $(1 / \mathrm{m})$ \\
\hline$\xi$ & coordinate variable defined in Eq. (9a) (-) \\
\hline$\sigma_{\mathrm{L}}$ & $\begin{array}{l}\text { parameter of linear fixed charge distribu- } \\
\text { tion }(-)\end{array}$ \\
\hline$\sigma_{\mathrm{s}}$ & $\begin{array}{l}\text { parameter of sinusoidal fixed charge dis } \\
\text { tribution }(-)\end{array}$ \\
\hline$\phi$ & angle coordinate $(-)$ \\
\hline$\chi$ & $\begin{array}{l}\text { scaled distance between the focus and the } \\
\text { center of an ellipse }(-)\end{array}$ \\
\hline$\psi$ & electrical potential $(\mathrm{V})$ \\
\hline $\bar{\psi}$ & scaled electrical potential $(-)$ \\
\hline
\end{tabular}

\section{References}

[1] J.A. Manzanares, W.D. Murphy, S. Mafe, H. Reiss, Numerical simulation of the nonequilibrium diffuse double layer in ionexchange membranes, J. Phys. Chem. 97 (1993) 8524.

[2] J. A Manzanares, S. Mafe, J. Pellicer, Transport phenomena and asymmetry effects in membranes with asymmetric fixed charge distributions, J. Phys. Chem. 95 (1991) 5620.

[3] C. Selvey, H. Reiss, Ion transport in inhomogeneous ion exchange membranes, J. Membr. Sci. 23 (1985) 23.

[4] H. Reiss, I.C. Bassignana, Critique of the mechanism of superselectivity in ion exchange membranes, J. Membr. Sci. 11 (1982) 219.

[5] J.A. Manzanares, S. Mafe, J. Pellicer, Current efficiency enhancement in membranes with macroscopic inhomogeneities in the fixed charge distribution, J. Chem. Soc. Faraday Trans. 88 (1992) 2355.

[6] V.A. Sokirko, J.A. Manzanares, J. Pellicer, The permselectivity of membrane systems with an inhomogeneous distribu- 
tion of fixed charge groups, J. Colloid Interface Sci. 168 (1994) 32.

[7] J.P. Hsu, K.L. Yang, Transport of ions through cylindrical ion-selective membranes, J. Phys. Chem. 100 (1996) 12503.

[8] A.P. Broek, H.A. Teunis, D. Bargeman, E.D. Sprengers, C.A. Smolders, Characterization of hollow fiber hemodialysis membranes: pore size distribution and performance, J. Membr. Sci. 73 (1992) 143.

[9] R.J. Hunter, Foundations of Colloid Science, Vol. 1, Oxford University Press, London, 1989.
[10] P. Moon, D.E. Spencer, Field Theory Handbook, Springer, Berlin, 1961

[11] N. Lakshminarayanaiah, Transport Phenomena in Membranes, Academic Press, New York, 1969.

[12] N. Lakshminarayanaiah, Equations of Membrane Biophysics, Academic Press, New York, 1969.

[13] R.P. Buck, Kinetics of bulk and interfacial ionic motion: microscopic bases and limits for the Nernst-Planck equation applied to membrance systems, J. Membr. Sci. 17 (1984) 1.

[14] C.F. Gerald, P.O. Wheatley, Applied Numerical Analysis, Addison-Wesley, Reading, MA, 1989. 